\title{
Untersuchungen über die Eigenhydrolyse von Cholinestern
}

\author{
Von W. Pilz und I. JohanN \\ Als dem Pbysiologisch-Chemischen und Analytischen Labor (Leiter: Dr. W. Pilz) der Ärztlichen Abteilung \\ (Leiter: Dr. H. Hörlein) der Farbenfabriken Bayer AG, Werk Leverkusen
}

(Eingegangen am 26. März 1966)

\begin{abstract}
Die Eigenhydrolyse von Acetylcholin, Benzoylcholin, Succinylbischolin, Succinylmonocholin und als Vergleichssubstanz von Benzoesäureäthylester-4-sulfosäure wird bei pH 7,4 über einen Zeitraum von $26 \mathrm{Stdn}$. bei $37^{\circ}$ verfolgt. Die Abhängigkeit der Verseifungsrate der erwähnten Ester innerhalb $24 \mathrm{Stdn}$. vom pH-Wert wird im Bereich von pH 6,4 bis pH 9,2 gemessen. Die Ergebnisse werden im Hinblick auf den Succinylbischolinabbau in vivo und insbesondere im Hinblick auf die Vielzahl der publizierten Methoden zur Bestimmung der Acetylcholinesteraseaktivität diskutiert.

The autohydrolysis of acetylcholine, benzoylcholine, succinylbischoline, succinylmonocholine and, as a standard, benzoic acid ethyl ester-4-sulphonic acid was measured over 26 hours at $\mathrm{pH} 7.4$ and $37^{\circ}$. The $\mathrm{pH}$-dependence of the rate of saponification of the above esters was measured in the range $\mathrm{pH} 6.4-9.2$ over 24 hours. The results are discussed in relation to the in vivo degradation of succinylbischoline and especially to the numerous published methods for the determination of acetylcholinesterase activity.
\end{abstract}

Im Zusammenhang mit dem umfangreichen Schrifttum über die In-vivo-Hydrolyse vom Succinylbischolin wurde bisher noch nie eine Untersuchung über die Eigenby'droly'se dieses Substrates durchgeführt. Auch im Hinblick auf die in großer Zahl publizierten und angewandten Methoden zur Bestimmung der Acetylcholinesteraseaktivität in biologischem Material ist es von Interesse, das Verhalten von Cholinestern und eine mögliche nicht enzymatische Verseifung derselben zu kennen.

Auch bei sehr diffizilen Methoden, die sich mit der Cholinesterspaltung durch menschliches Serum und mit der Definition von Kennzahlen $(1,2)$ beschäftigen, wird in der Mehrzahl der Fälle die Notwendigkeit der Messung einer Eigenverseifung der Substrate (meist Benzoylcholin) außer acht gelassen, oder wenigstens nicht erwähnt $(3,4,5)$. Da es eine Vielzahl von Meinungen über den Abbau von Succinylbischolin und die damit in Zusammenhang stehenden Probleme der nach Succinylbischolinapplikation gelegentlich auftretenden verlängerten Apnoe gibt und außerdem in der Mehrzahl der Bestimmungsmethoden für die Serumacetylcholinesterase und Serumaliesterase, die kaum noch überschaubar sind, in vielen Fällen die Eigenverseifung der Substrate nicht berücksichtigt wird, war eine systematische Untersuchung über die Eigenhydrolyse von Cholinestern in Abhängigkeit von der Zeit und vom $\mathrm{pH}$-Wert von aktuellem Interesse.

\section{Methodik}

In die Untersuchung wurden folgende Cholinester einbezogen: Acetylcholin, Benzoylcholin, Succinylbischolin, Succinylmonocholin und als Vergleichssubstanz ein Ester, dessen Alkoholkomponente nicht Cholin war; wegen der güten Wasserlöslichkeit entschieden wir uns für dịe Benzoesäureäthylester-4-sulfosäure. Die Esterlösungen wurden über $24 \mathrm{Stdn}$. bei $37^{\circ}$ und $\mathrm{pH} 7,4$ bebrütet und in definierten Zeitabständen aliquote Anteile entnommen. Die Substratkonzentration war in allen Fällen $8 \cdot 10^{-3}$ Ãq/l. Die erste Entnahme erfolgte stets unmittelbar nach der Herstellung. Die Ester wurden als komplexe Ferrihydroxamate (6) bestimmt. In einer weiteren Untersuchung wurden Esterlösungen $24 \mathrm{Stdn}$. bei $\mathrm{pH}$-Werten $z$ wischen 6,3 und 9,5 bebrütet und nach Ablauf dieser Zeit die noch vorhandene Estermenge nach (6) gemessen. Als Puffer dienten ausschließlich Tris-Essigsäure-Puffer (7). In allen Fällen wurde die Anfangskonzentration gleich $100 \%$ ge- setzt und die nach der Bebrütung noch vorhandene Estermenge in $\%$ angegeben.

\section{Ergebnisse}

Der zeitliche Ablauf der Eigenhydrolyse bei $\mathrm{pH} 7,4$ und $37^{\circ}$ ist in Abbildung 1 dargestellt. Wie ersichtlich, zeigen Acetylcholin und Benzoylcholin einen ähnlichen Kurvenverlauf, während Succinylmonocholin sich völlig anders verhält. Der Verseifungsverlauf von Succinylbischolin folgt einer S-förmigen Kurve und weicht damit vom Verhalten der anderen Ester deutlich ab. Dagegen ist die Eigenverseifung der Benzoesäureäthylester-4-sulfosäure durch eine Gerade darstellbar.

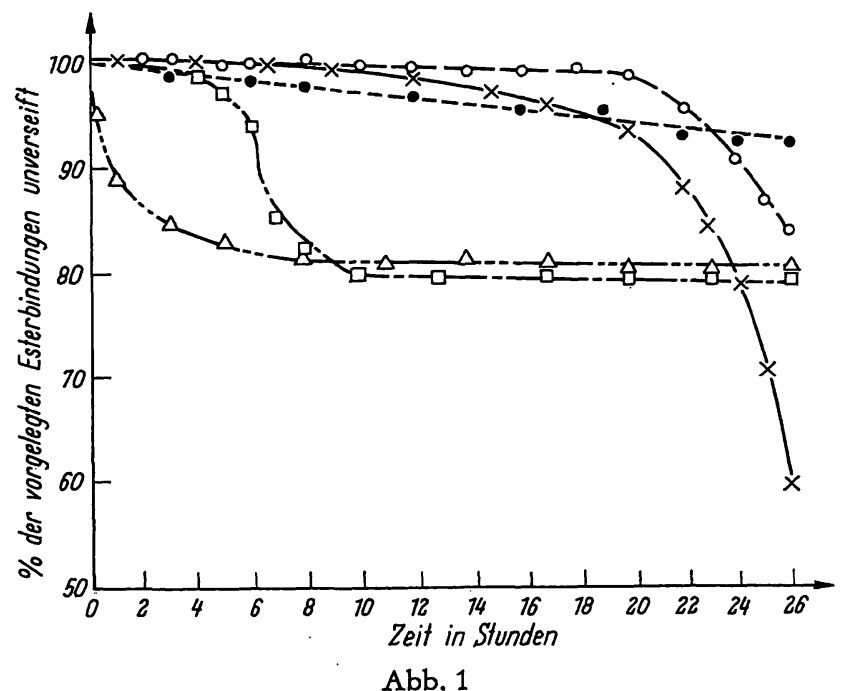

Eigenverseifung von 4 Cholinestern und eines Vergleichsesters in Abhängigkeit von der Zeit

Ansätze: $8 \cdot 10^{-3} \AA \ddot{q} / l$ in TRIS-Essigsäurepuffer $\mathrm{pH} 7,4 ; 37^{\circ}$; $x \rightarrow \times$ Acetylcholin; O-..- O Benzoylcholin; $\square--\square$ Succinylbischolin; $\triangle-.-\triangle$ Succinylmonocholin; -..- Benzoesäureäthylester-4-sulfosaures Kalium;

Abszisse: Zeit in Stdn.; Ordinate: \% Ester unverseift

Bei einer Verseifungszeit von 24 Stdn. ist die Menge des unverseiften Esters bei Succinylbischolin und Succinylmonocholin linear vom $\mathrm{pH}$-Wert abhängig. Diese beiden Cholinester werden in einer wesentlich größeren Rate als die als Vergleich herangezogene Benzoesäureäthylester-4-sulfosäure gespalten (Abb. 2). 


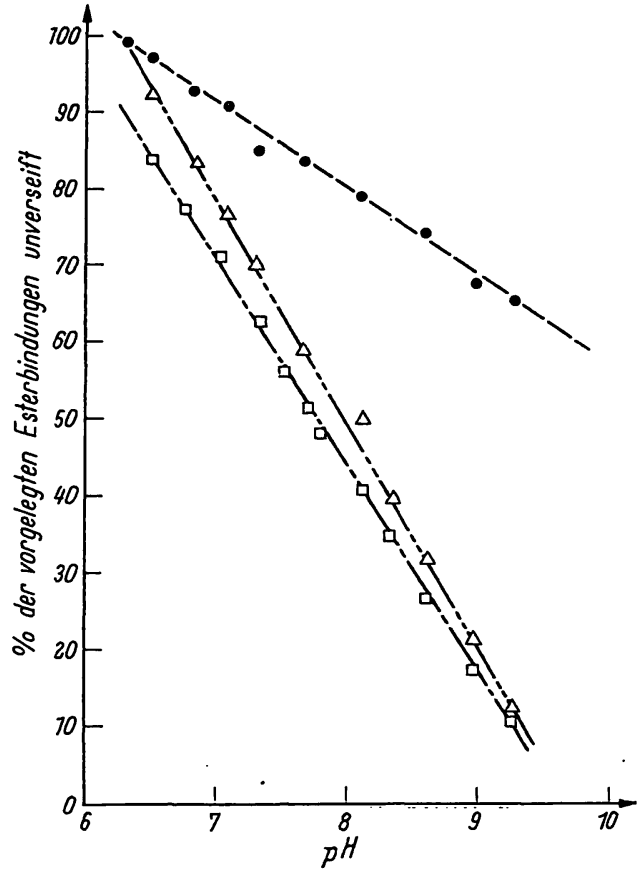

Abb. 2

Eigenverseifung von 2 Cholinestern und eines Vergleichsesters in Abhängigkeit vom $\mathrm{pH}-\mathrm{W}^{\prime}$ ert.

Ansatz: $8 \cdot 10^{-3} \AA \AA \mathrm{q} / l, 37^{\circ}$, TRIS-Essigsäurepuffer; Werte nach 24 Stdn.; $\square--\square$ Succinylbischolin; $\triangle-\cdots \triangle \triangle$ Succinylmonocholin; -..-- Benzoesäureäthylester-4-sulfosaures Kalium; Abszisse: $\mathrm{pH}-\mathrm{W}$ erte; Ordinate: \% Ester unverseift

Ganz anders verhalten sich Acetylcholin und Benzoylcholin (Abb. 3). Die Kurve ähnelt in erster Näherung einer Titrationskurve, deren Äquivalenzpunkt in der Gegend von $\mathrm{pH} 8$ liegt.

Wegen dieser unerwarteten Resultate, insbesondere wegen des so stark unterschiedlichen Verhaltens von Acetylcholin und Benzoylcholin auf der einen Seite und den beiden Succinylcholinestern auf der anderen Seite, wurden die beschriebenen Untersuchungen mehrmals wiederholt, wobei stets die gleichen Ergebnisse erhalten wurden.

\section{Diskussion}

Das wesentlichste Ergebnis ist die Tatsache, daß sich Cholin als Alkoholkomponente enthaltende Ester bei der Eigenhydrolyse anders als gewöhnliche Ester verhalten. Bei der physiologischen Bedeutung der Cholinester können daraus manche Schlüsse gezogen werden. Außerdem ist der Reaktionsmechanismus bei den vier untersuchten Estern offenbar verschieden.

Bei physiologischem pH-Wert setzt die Eigenhydrolyse von Succinylmonocholin am schnellsten ein. Succinylbischolin ergibt eine S-förmige Kurve, die unter Um- . ständen die von KALOw (8) aufgefundene Hydrolyse des Esters durch menschliches Serum erklären könnte. Es kann danach nicht ausgeschlossen werden, $\mathrm{da} ß$ diese Befunde wenigstens teilweise auf einer Eigenhydrolyse des Succinylbischolins beruhen. Das steht im Einklang mit den von uns erhobenen Befunden $(9,10)$, daß weder Succinylmonocholin noch Succinylbischolin vom menschlichen Serum gespalten werden. Es ist durchaus

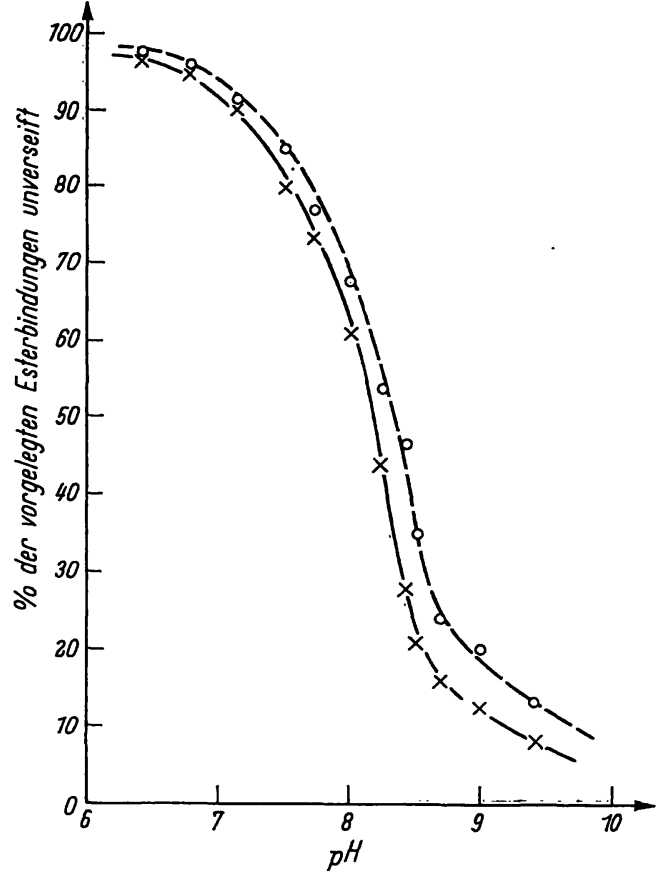

Abb. 3

Eigenverseifung von Acetylcholin und Benzoylcholin in Abhängigkeit vom pH-Wert

Ansatz: $8 \cdot 10^{-3} \AA \AA \mathrm{q} / l, 37^{\circ}$, TRIS-Essigsäurepuffer; Werte nach 24 Stdn.; $x \longrightarrow \times$ Acetylcholin; O-.- O Benzoylcholin; Abszisse: pH-Wert; Ordinate: \% Ester unverseift

möglich, daß appliziertes Succinylbischolin mit dem strömenden Blut Stellen passiert, an denen höhere pH-Werte als 7,4 herrschen (z. B. (11)). Dadurch würde die Eigenhydrolyse sehr viel schneller erfolgen, als aus den in Abbildung 1 dargestellten Befunden zu erwarten wäre. Es ist denkbar, daß der Abbau von Succinylbischolin und die damit verbundene Beendigung der Relaxierung qu einem Teil auf der Eigenhydrolyse des Esters beruhen. Der Hauptanteil der Hydrolyse von Süccinylbischolin zu Succinylmonocholin erfolgt in vivo jedoch enzymatisch in der Lunge (12) (vgl. auch (13)). Die vorgelegten Ergebnisse haben schwerwiegende Folgen für die Bewiertung der Vielzahl der gebräuchlichen Methoden zur Bestimmung der Acetylcholinesteraseaktivität im menschlichen Blut. Wir selbst konnten vor kurzem zeigen (14), daß das pH-Optimum der Acetylcholinesterasen im Vollblut bei $\mathrm{pH}$ 8,6 liegt, während in der Literatur für das Serumferment ein pH-Optimum von 8,0 bis 8,5 (15) und für das Enzym der Erythrozyten von 7,5 bis 8 (16) angegeben wird (vgl. dagegen (17)). Arbeitet man bei diesen $\mathrm{pH}$-Werten und nimmt auf die Eigenverseifung der Substrate keine Rücksicht, kann man zu groben Fehlresultaten gelangen. Insbesondere jene Methoden, die auf einer Änderung des elektrometrisch gemessenen $\mathrm{pH}$-Wertes beruhen (18), gestatten praktisch keine Kontrolle bei Eigenverseifung der verschiedenen Substrate. Besonders problematisch sind Ergebnisse zu beurteilen, die mit Methoden erhalten werden, bei denen die ursprüngliche elektrometrische Methode (18) zu einer Mikromethode modifiziert wurde (19). Es gibt Methoden auf dieser Basis; die 
hauptsächlich in den USA gebräuchlich sind, bei denen das $\Delta \mathrm{pH} / \mathrm{Std}$. nur 0,2 $\mathrm{pH}$-Einheiten (mit einem angeblichen Fehler von $\pm 0,02$ bis $0,03 \Delta \mathrm{pH} / \mathrm{Std}$.) angegeben wird (20). Eine der gebräuchlichsten Methoden stammt von RIDER und Mitarbeitern (21). Es ist unschwer einzusehen, daß die Eigenverseifung der verwendeten Substrate als Fehler in das Resultat eingeht.

Eine weitere, besonders in den USA sehr verbreitete Schnellmethode beruht auf der Tatsache, daß die enzymatisch aus den jeweils verwendeten Cholinestern in Freiheit gesetzten Säuren einen pH-Abfall bewirken, der mit Hilfe eines Indikators angezeigt wird (22). Meist wird die Zeit vom Zusammenbringen des biologischen Materials mit dem Substrat bis zum Umschlag des
Indikators gemessen und als Maß für die Cholinesteraseaktivität bewertet. Ein ähnliches Verfahren wurde 1959 auch in Europa empfohlen, konnte sich aber nicht recht durchsetzen (23). Bei den meisten der erwähnten Methoden geht die Eigenverseifung der Substrate als Enzymaktivität in das Resultat ein. Dagegen geht bei allen von uns bekanntgegebenen Methoden und deren Varianten (z. B. (10,14, 24)) die Eigenverseifung der Substrate nicht in das Resultat ein. Die gefundenen Ergebnisse sind unverfälschte Enzymaktivitäten.

Welche Bedeutung der Eigenhydrolyse der Cholinester bei der Übertragung von Impulsen zwischen Nervenendplatten und Muskel zukommen, muß weiteren Untersuchungen vorbehalten bleiben.

\title{
Literatur
}

1. Goedde, H. W., D. Gehring und R. Hofmann, Z. analyt. Chem. 212, 238 (1965). - 2. Kalow, W. und K. Genest, Canad. J. Biochem. Physiol. 35, 339 (1957). - 3. Harris, H. und M. WhitTAKER, Ann. hum. Genet. 26, 59 (1962). - 4. Harris, H. und M. Whittaker, Nature (London) 191, 4787 (1961). - 5. Hofstee, B. H., Science (Lancaster, Pa.) 114, 128 (1951). - 6. Pruz, W., Z. analyt. Chem., 162, 81 (1958); 166, 189 (1959). - 7. PrLz, W. und I. Johann, Z. analyt. Chem. 315, 105 (1965). - 8. Kalow, W., Anesthesiology 20, 505 (1959). - 9. Pilz, W. und H. HörLein, Hoppe-Seyler's Z. physiol. Chem. 339, 157 (1964). - 10. Przz, W., diese Z. 3, 89 (1965). - 11. Prlz, W., H. Hörlein und E. Stelzr, Hoppe Scylers' Z. physiol. Chem., im Druck. - 12. Przz, W., Biochem. Z., im Druck. - 13. Doenicke, A., Dtsch. med. Wschr., im Druck. - 14. Pilz, W., I. Johann und E. Stelzz, Klin. Wschr. 43, 1227 (1965). - 15. Eassohn, L. H. und E. Stedman, Proc. Roy. Soc. (London) Ser. B 121, 142 (1936). - 16. Alles, G. A. und R. C. Hawes, J. biol. Chemistry 133, 375 (1940). - 17. Pilz, W., Hoppe-Seyler's Z. physiol. Chem., im Druck. - 18. Mrchex, H. O., J. Laborat. Clin. Med., S. Louis 34, 1564 (1949). - 19. FRYER,
J. H., R. G. D. Steer und H. H. Williams, Arch. industr. Health 12, 406 (1955). Stubbs, J. L. und J. I. FAbes, Amer. J. Med. Techn. 26, 25 (1960). Wolfsie, J. H. und G. D. Winter, Arch. industr. Hyg. 6, 43 (1952). - 20. Gammin, J. F., B. B. Brody und F. A. Fucrle, Medical Invest, Branch Scientific Report Nr. 3, Dugway Proving Ground, Utah, July 14, (1954). - 21. Rider, J.A., J. L. Hodges, J. Swader und A. D. Wiggins, J. Laborat. Clin. Med., S. Louis 50, 376 (1957). - 22. Davies, D. R. und J. D. Nicholls, Brit. Med. J. 1, 1373 (1955). Edson, E. F., World Crops, 10, 49 (1958). Edson, E. F. und M. L. Fenwick, Brit. Med. J. 1, 1218 (1955). Fleisher, J. H., G. S. Woodson und L. Simet, Arch. industr. Health 14, 510 (1956). Limperos, G. und K. E. Ravta, Science (Washington) 117, 453 (1953). - 23. SAILER, S. und $H$. Braunstenner, Klin. Wschr. 37, 986 (1959). - 24. Pilz, W., Klin. Wschr. 36, 1017 (1958). Prlz, W., Zschr. exper. Med. 132, 310 (1959). Pruz, W., in: Handbuch der enzymatischen Analyse, Verlag Chemie, Weinheim/Bergstr. (1962). Pilz, W. und G. Kimmerle, Hoppe-Seyler's Z. physiol. Chem. 327, 280 (1962), vgl. auch $(10,12)$.
Dr. W. Pilz

Leiter des Physiol.-Chem. und Analyt. Labors der ärztlichen Abt. 509 Leverkusen-Bayerwerk

\section{Plasmabogen für klinisch-chemische Analysen ${ }^{1)}$}

\author{
Von R. Herrmann und K. Rötger \\ Aus der Abteilung für Medizinische Phjsite an der Universitäts-Hautklinik Gießen (Leiter: Prof. Dr. R. Herrmann)
}

(Eingegangen am 21. März 1966)

\begin{abstract}
Einleitend werden die Schwierigkeiten aufgezeigt, die zu überwinden sind, wenn man mit einfachen und schnellen flammenspektrophotometrischen Analysenmethoden möglichst viele Elementkonzentrationen in biologischen Lösungen analysieren will. Bei den schwerer dissoziierbaren Bestandteilen ist die Anwendung größerer Energien bzw. höherer Temperaturen nötig. Hierfür bietet sich der Plasmabogen an. Es wird eine bei Untersuchungen an biologischen Lösungen bewährte Modifikation eines geeigneten Brenners beschrieben und es werden damit bestimmte chemische Nachweisgrenzen angegeben.

If a simple and rapid flame photometric method is required for the determination of as many elements as possible in biological fluids, certain difficulties must be overcome; these are discussed. With the less dissociable components, greater energies or higher temperatures are necessary. The plasma arc can be used for this purpose. A modified burner, which can be used for studies on biological solutions, is described and its limits of chemical detection are given.
\end{abstract}

Emissionsflammenphotometrische Analysenmethoden haben sich für die Bestimmung der Elementkonzentrationen von Natrium, Kalium und Calcium in Körper-

1) Unterstützt mit Mitteln der Deutschen Forschungsgemeinschaft. flüssigkeiten dank ihrer Einfachheit, Schnelligkeit, Spezifität, geringer Streubreite und ihrer geringen Störantälligkeit in den klinisch-chemischen Laboratorien weitgehend durchgesetzt (1). Die Vorzüge dieser 